\title{
Consenso AURORA: risco cardiovascular na pessoa com diabetes mellitus tipo 2
}

Tiago Maricoto, ${ }^{1}$ José Pedro Antunes, ${ }^{2}$ Manuel Rodrigues Pereira, ${ }^{3}$ Susana Corte-Real, ${ }^{4}$ Tiago Taveira-Gomes, ${ }^{5}$ João Pedro Nobre ${ }^{6}$

\section{RESUMO}

Objetivo: Desenvolver um documento de consenso orientado para a prática clínica no contexto da medicina geral e familiar relativamente à gestão farmacológica do risco cardiovascular na pessoa com diabetes mellitus tipo 2.

Métodos: Foi reunido um painel de seis especialistas em medicina geral e familiar que desenvolveu uma revisão bibliográfica não sistemática de recomendações clínicas, meta-análises e estudos originais e de revisão relativos ao controlo dos principais parâmetros de risco cardiovascular no adulto com diabetes mellitus tipo 2. Foi analisada e debatida de modo exaustivo a evidência identificada que foi posteriormente sumarizada num conjunto de recomendações práticas.

Resultados: Foram identificadas 23 fontes bibliográficas, entre recomendações clínicas, estudos originais, meta-análises e estudos secundários com base nos quais se elaboraram 13 recomendações relativas à terapêutica com antidiabéticos, cinco recomendações relativas à terapêutica com anti-hipertensores, cinco recomendações relativas à terapêutica antidislipidémica e 10 recomendações relativas à terapêutica com antiagregantes plaquetários.

Conclusão: A gestão do risco cardiovascular e da insuficiência cardíaca na pessoa com diabetes mellitus tipo 2 constitui um exercício clínico desafiante que requer uma análise holística e individualizada de cada pessoa. Novas armas farmacológicas estão agora disponíveis e devem ser utilizadas de forma incisiva para garantir um controlo adequado da glicemia e dos fatores de risco cardiovascular, minimizando a morbimortalidade inerente às complicações da diabetes mellitus tipo 2.

Palavras-chave: Diabetes mellitus tipo 2; Medicina geral e familiar; Risco cardiovascular.

\section{INTRODUÇÃO}

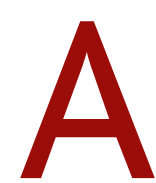

diabetes mellitus tipo 2 (DM2) é uma doença metabólica complexa envolvendo múltiplos mecanismos fisiopatológicos. Estima-se que em Portugal a prevalência global seja de $13,3 \%$, representando mais de um milhão de portugueses, mas muitos destes doentes ainda estão por diagnosticar. ${ }^{1-2}$ Diversas comorbilidades estão associadas à DM2, das quais se destacam a obesidade e o excesso de peso, presente em quase todos estes doentes, ${ }^{1}$ a hipertensão arterial (HTA), presente em cerca de metade dos casos, ${ }^{3}$ bem como a dislipidemia e a doença cardiovascular aterosclerótica, também presente na

1. USF Aveiro-Aradas, ACeS Baixo Vouga. Faculdade de Ciências da Saúde, Universidade da Beira Interior.

2. USF Viva Saúde, ACeS do Baixo Vouga.

3. UCSP de Alcochete, ACeS Arco Ribeirinho.

4. USF S. Julião, ACeS Lisboa Ocidental e Oeiras.

5. Faculdade de Medicina, Universidade do Porto.

6. USF Rodrigues Miguéis, ACeS Lisboa Norte. maioria dos casos, ${ }^{4-5}$ Trata-se de uma doença que pode contribuir para inúmeros desfechos adversos, como a progressão da doença renal crónica (DRC), ${ }^{6}$ insuficiência cardíaca (IC), ${ }^{7}$ enfarte agudo do miocárdio (EAM) e acidente vascular cerebral (AVC).$^{8-9}$ A DM2 está associada a cerca de $3,8 \%$ das mortes ocorridas em Portugal e, apesar da sua morbilidade ter vindo a diminuir nos últimos anos, representa cerca de $1 \%$ do PIB e $10 \%$ do total da despesa nacional em saúde. ${ }^{1}$

A DM2 reduz a esperança média de vida em cerca de 10 anos e a principal causa de morte nestes doentes são as doenças cardiovasculares (DCV), estimando-se que mais de dois terços das pessoas com DM2 acima dos 65 anos venham a morrer de alguma forma de DCV. Por outro lado, a DM2 cruza-se com as doenças cardiovasculares a todos os níveis e tem fortes implicações no desenvolvimento e progressão da DCV. Desde o Framingham Heart Study, ${ }^{10}$ entre outros estudos seminais, sabe-se que a DM2 é um importante fator de risco 
cardiovascular (RCV), agravando substancialmente o risco de EAM, IC, doença arterial periférica (DAP) e AVC. Além disso, nos doentes com DCV estabelecida a DM2 é um fator de prognóstico adverso. ${ }^{10} \mathrm{Em} 1998$, Haffner e colaboradores mostraram que a incidência de EAM em doentes com DM2 é duas a cinco vezes maior do que em doentes sem DM2, ${ }^{11}$ o que levou a DM2 a ser considerada um equivalente de DCV estabelecida ou EAM prévio, enfatizando a importância da aterosclerose na DM2.

Neste sentido, o foco das orientações clínicas dirigidas ao tratamento da DM2 foi evoluindo, passando da ênfase no controlo glicémico para a prevenção das complicações da DCV.Tem vindo a ser aconselhado um controlo cada vez mais agressivo dos fatores de RCV e apontando como alvo glicémico aquele que minimiza as complicações de DCV e de mortalidade. ${ }^{1-2}$

A relação entre a DM2 e a doença aterosclerótica levou a uma valorização pronunciada das complicações micro e macrovasculares da DM2 em comparação com a associação entre a IC e a DM2. De facto, desde 1979 que os dados do Framingham Heart Study sugerem que a DM2 duplica o risco de IC nos homens e quintuplica este risco nas mulheres, uma discrepância de género que ainda não é completamente compreendida. ${ }^{10} \mathrm{~A}$ IC é um dos marcadores de prognóstico mais adverso nos doentes com DCV estabelecida, estando a concomitância de IC e DM2 associada a um pior estado funcional e a um aumento da mortalidade, nomeadamente por DCV e por todas as causas, quer em doentes com fração de ejeção reduzida quer com fração de ejeção preservada. ${ }^{12}$

A DM2 associa-se a uma prevalência elevada de disfunção ventricular esquerda, tanto diastólica como sistólica, e acelera o desenvolvimento de IC. Para além dos preditores cardíacos de IC, como a disfunção ventricular esquerda, a hipertrofia ventricular esquerda e a doença coronária, a probabilidade de desenvolvimento de IC aumenta com um mau controlo glicémico, com a duração da DM2, com o tratamento com insulina e com a presença de complicações microvasculares. ${ }^{12}$ Os resultados de múltiplos ensaios clínicos aleatorizados bem como diversos estudos observacionais demonstram que a IC é quase tão comum como as complicações isquémicas nos doentes com DM2, permanecendo como uma das principais causas de internamento hospitalar nesta população..$^{1-2,12}$ Enquanto o controlo dos fatores de RCV convencionais (como a
HTA, o tabagismo e a dislipidemia) reduz a incidência das complicações isquémicas, a IC continua a ser um problema sem terapêutica específica na DM2, para o qual o controlo glicémico intensivo tem mostrado conferir pouco benefício.

O tratamento da DM2 passa pela adoção de medidas de estilo de vida saudáveis e, na maioria dos casos, por instituição concomitante de terapias farmacológicas. ${ }^{13}$ Têm surgido múltiplas opções terapêuticas nos últimos anos endereçadas aos diferentes mecanismos fisiopatológicos intervenientes no desenvolvimento de DM2. ${ }^{14} \mathrm{O}$ seu uso deve considerar o equilíbrio entre a eficácia na redução da glicemia, os benefícios no controlo da DCV, os efeitos secundários, os custos e as preferências individuais da pessoa. ${ }^{13} \mathrm{~A}$ metformina mantém-se como o fármaco de eleição para o tratamento inicial da DM2; ${ }^{15}$ no entanto, é frequente a associação ou substituição por outros antidiabéticos, seja por não atingimento dos alvos terapêuticos, intolerância ou contraindicação. No que respeita às restantes classes farmacológicas destaca-se, desde 2008, a avaliação da eficácia e segurança dos inibidores do cotransportador de sódio e glicose 2 (iSGLT-2) e dos agonistas do recetor do glucagon-like peptide 1 (arGLP-1). Na generalidade, os resultados têm apontado para a superioridade de alguns destes fármacos na redução do risco de eventos cardiovasculares adversos e de mortalidade. ${ }^{16-17}$ Este fator levou a várias atualizações recentes das principais recomendações internacionais, que colocam estas classes como opção preferencial em casos em doentes com ou sem DCV estabelecida. ${ }^{13,18-20}$ Adicionalmente, alguns destes fármacos têm efeitos benéficos, nomeadamente na redução de peso, tanto os iSGLT2 como os arGLP1, ou na diminuição da pressão arterial e na proteção renal, no caso dos iSGLT2. Em relação a estes últimos, a partir dos primeiros ensaios clínicos ${ }^{21-23} \mathrm{di}$ versos outros estudos e subanálises têm demonstrado os benefícios desta classe na redução do risco de mortalidade cardiovascular global, redução de internamentos e mortalidade por IC. No que respeita aos arGLP-1, os principais estudos demonstraram resultados heterogéneos, revelando alguns potenciais benefícios na mortalidade cardiovascular global e cardio-renal. ${ }^{24,26}$ Outros estudos têm ainda revisitado o papel da terapêutica antitrombótica em prevenção primária de eventos em pessoas com DM2, idosos e pessoas com 
fatores de $\mathrm{RCV}^{8-9,27,28}$ e novos estudos têm abordado o tratamento destes doentes mesmo após ocorrerem eventos coronários agudos, sugerindo novos protocolos de atuação farmacológica à prática clínica. ${ }^{29-30}$

Já em relação ao tratamento farmacológico da dislipidemia e hipertensão arterial não têm surgido novos fármacos recentes e, apesar de novas evidências terem surgido sobre a eficácia de algumas opções terapêuticas e associações fixas sobre os principais outcomes, poucas alterações têm surgido recentemente.

O médico de família desempenha um papel central na gestão da DM2. Assim, pretende-se sumarizar, de modo orientado para a prática clínica, a evidência relativa à gestão do RCV e da IC na pessoa com DM2, integrando orientações relativas à terapêutica antidiabética, antidislipidémica, anti-hipertensora e antitrombótica.

\section{MÉTODOS}

Foi reunido um painel de seis especialistas em medicina geral e familiar que desenvolveram o trabalho em três fases, que se descrevem seguidamente.

Na primeira fase realizou-se uma pesquisa bibliográfica não sistemática utilizando as bases de dados MEDLINE e Central com os termos livres "Diabetes mellitus tipo 2", "risco cardiovascular”, "anti-agregação", "terapêutica antitrombótica", "hipertensão arterial", "síndrome coronário agudo" "dislipidemia" e seus respetivos equivalentes MeSH, nomeadamente Diabetes Mellitus, Cardiovascular Diseases, Platelet Aggregation Inhibitors, Hypertension, Acute Coronary Syndrome e Dyslipidemias. Foram consideradas orientações clínicas, meta-análises, revisões sistemáticas e estudos originais relativos à população adulta.

Na segunda fase, os estudos foram analisados e debatidos de modo exaustivo pelo painel de modo a alcançar consenso na interpretação da evidência científica e das recomendações publicadas, à luz da prática clínica em medicina geral e familiar.

$\mathrm{Na}$ terceira fase foram desenvolvidas por consenso as recomendações seguidamente apresentadas, que sumarizam os principais aspetos a considerar na gestão do RCV e da IC na DM2, e elaborado um diagrama que representa as mesmas de um modo integrado.

\section{RESULTADOS}

Da pesquisa bibliográfica inicial, e após análise do painel de peritos, recolheram-se 23 fontes bibliográficas relevantes, entre normas de orientação e guidelines, meta-análises e estudos originais adicionais, que sustentam as principais recomendações elaboradas. A Figura 1 resume as principais recomendações na abordagem do RCV da pessoa com DM2 e suas principais comorbilidades e complicações.

\section{Recomendações para terapêutica antidiabética}

1. Para além do controlo glicémico, deverão ser privilegiadas terapêuticas que permitam uma abordagem multifatorial no tratamento da DM2. ${ }^{13,18-20}$

2. De modo a maximizar a adesão à terapêutica, a abordagem farmacológica deve ser discutida e acordada com o doente e individualizada para o seu perfil. ${ }^{13,18-20}$

3. Deve privilegiar-se a utilização de fármacos com perfil de efetividade e segurança favoráveis, nomeadamente com baixo risco de hipoglicemia e de aumento ponderal. ${ }^{13,18-20}$

4. A metformina continua a ser a primeira linha farmacológica no tratamento da DM2. ${ }^{13,18-20}$

5. Nas pessoas com DM2 e fatores de RCV e que não atingem o alvo glicémico com metformina em monoterapia deve ser considerada terapêutica adicional com um iSLGT-2 ou arGLP- $1 .^{31}$

6. Na pessoa com DM2, com DCV aterosclerótica estabelecida ou IC, ou DRC, que esteja controlada com terapêutica dupla antidiabética sem benefício cardiovascular, é recomendada a substituição do fármaco de $2^{\text {a }}$ linha por uma das classes que tenha demonstrado benefício cardiovascular: iSGLT-2 , caso a função renal o permita, ou arGLP- $1 .^{13,18-20}$

7. Os inibidores da DPP-4 devem ser considerados no idoso frágil como terapêutica de $2^{\mathrm{a}}$ linha. ${ }^{13,18,32}$

8. Os iSGLT-2 disponíveis em Portugal reduzem o risco de EAM, AVC e morte por causa cardiovascular em indivíduos com DCV estabelecida. ${ }^{21-22,33-34}$

9. Os arGLP-1, nomeadamente dulaglutido, liraglutido e semaglutido, reduzem o risco de EAM, AVC e morte por causa cardiovascular. ${ }^{17}$

10. Os iSGLT-2 constituem uma importante terapêutica para a prevenção das hospitalizações por IC na pessoa com DM2. Este efeito parece não estar dependente do RCV ou IC prévios. Este benefício mantém-se em diferentes espectros de perfil 


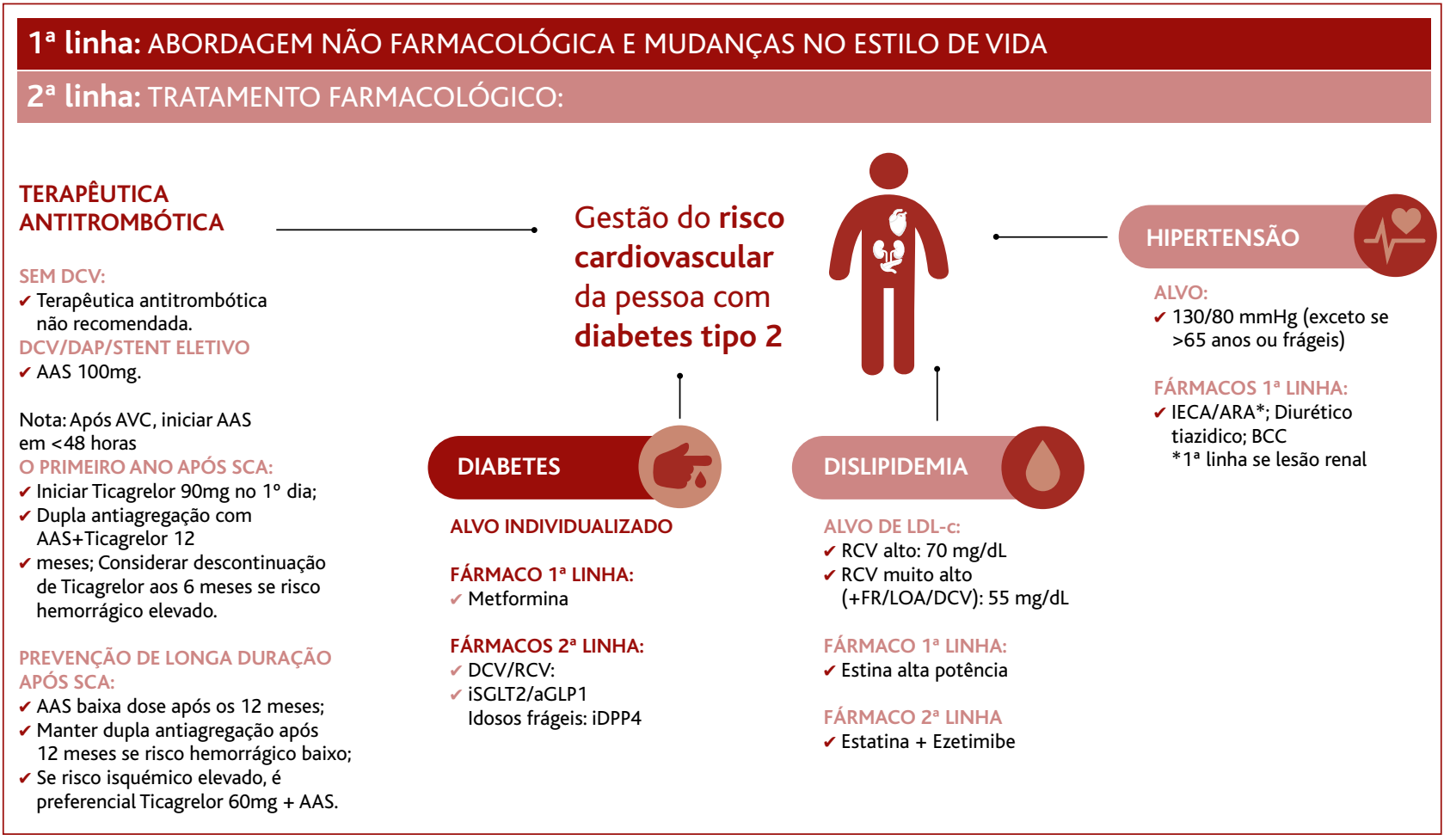

Figura 1. Diagrama resumo das recomendações práticas na gestão do risco cardiovascular do doente com diabetes tipo 2 e suas principais comorbilidades.

AAS - Ácido acetilsalicílico; ARA - Antagonista dos recetores da angiotensina; AVC - Acidente vascular cerebral; BCC - Bloqueador dos canais de cálcio; DAP - Doença arterial periférica; DCV - Doença cardiovascular; IECA - Inibidor da enzima de conversão da angiotensina; LOA - Lesão de órgão-alvo; RCV - Risco cardiovascular; SCA - Síndroma coronária aguda.

cardiovascular da pessoa com DM2 (Tabelas 1 e 2). ${ }^{16}$

11. Independentemente da função renal, os iSGLT-2 demonstraram reduzir a progressão da DRC em doentes com e sem DCV estabelecida. ${ }^{35-37}$

12. Em doentes que apresentem DRC (albuminúria $>30 \mathrm{mg} / \mathrm{g}$ e/ ou diminuição da TFGe $<90 \mathrm{~mL} / \mathrm{min} /$ $/ 1,73 \mathrm{~m}^{2}$ ) deve ser privilegiada a instituição de iSGLT- $2{ }^{38}$ considerando as características do medicamento.

13. As associações fixas de fármacos antidiabéticos constituem uma importante ferramenta para promover a adesão à terapêutica. ${ }^{39}$

\section{Recomendações para terapêutica anti-hipertensora}

1. Na pessoa com DM2, HTA e DCV estabelecida ou doente de muito alto RCV, o objetivo clínico de pressão arterial é < 130/80mmHg, se alcançado com segurança, ${ }^{18,40}$ mas não < 120/70; nos indivíduos com mais de 65 anos o objetivo de pressão arterial sis- tólica deverá ser 130-139mmHg, mas nos idosos frágeis os valores-alvo poderão ser mais elevados e definidos caso a caso para evitar iatrogenia. ${ }^{40}$

2. O tratamento da HTA na pessoa com DM2 deve incluir classes farmacológicas que demonstraram reduzir eventos cardiovasculares neste perfil de doentes, concretamente os inibidores da enzima conversora da angiotensina, os antagonistas dos recetores da angiotensina II, diuréticos (e.g., tiazídicos ou tiazide-like) ou antagonistas dos canais de cálcio. ${ }^{18,40}$

3. A terapêutica de primeira linha no tratamento da hipertensão arterial na pessoa com DM2 e DRC deverá ser um inibidor da enzima conversora da angiotensina ou um antagonista de recetores da angiotensina II. Quando necessário, deve ser considerada uma associação com bloqueador dos canais de cálcio para atingir o alvo terapêutico. ${ }^{40}$

4. Quando os alvos de pressão arterial não estão a ser alcançados com três classes anti-hipertensoras, 


\begin{tabular}{|c|c|c|c|}
\hline Variável & EMPA-REG OUTCOME & Programa CANVAS & DECLARE TIMI 58 \\
\hline Fármaco & Empagliflozina & Canagliflozina & Dapagiflozina \\
\hline População & 7020 & 10142 & 17160 \\
\hline Mediana de seguimento (anos) & 3,1 & 3,6 & 4,2 \\
\hline Doença cardiovascular aterosclerótica conhecida & 99 & 66 & 41 \\
\hline Insuficiência renal & 26 & 20 & 7 \\
\hline Sexo feminino & 29 & 36 & 37 \\
\hline Utilização de insulina no início do estudo & 48 & 50 & 41 \\
\hline HbA1c (\%) & $8,1 \pm 0,8$ & $8,7 \pm 1,5$ & $8,3 \pm 1,2$ \\
\hline Taxa de evento no grupo placebo (\%/ano) & 4,4 & 3,2 & 2,4 \\
\hline
\end{tabular}

Nota: Adaptado de Home P. Diabetologia 2019. https://doi.org/10.1007/s00125-018-4801.

\section{TABELA 2. Principais resultados dos estudos de segurança cardiovascular com iSGLT-2}

\begin{tabular}{|c|c|c|c|c|c|c|}
\hline Estudo & Tratamento & 3P - MACE & Morte CV & $\begin{array}{l}\text { Mortalidade por } \\
\text { todas as causas }\end{array}$ & $\begin{array}{c}\text { Hospitalização } \\
\text { por IC }\end{array}$ & $\begin{array}{c}\text { Marcador de } \\
\text { lesão renal }\end{array}$ \\
\hline EMPA-REG OUTCOME & Empagliflozina & $\begin{array}{c}0,86 \\
(0,74,0,99)\end{array}$ & $\begin{array}{c}0,62 \\
(0,49,0,77)\end{array}$ & $\begin{array}{c}0,68 \\
(0,57,0,82)\end{array}$ & $\begin{array}{c}0,65 \\
(0,50,0,85)\end{array}$ & $\begin{array}{c}0,54 \\
(0,40,0,75)\end{array}$ \\
\hline CANVAS Program $^{c}$ & Canagliflozina & $\begin{array}{c}0,86 \\
(0,75,0,97)\end{array}$ & $\begin{array}{c}0,90 \\
(0,71,1,15)\end{array}$ & $\begin{array}{c}0,87 \\
(0,74,1,01)\end{array}$ & $\begin{array}{c}0,67 \\
(0,52,0,87)\end{array}$ & $\begin{array}{c}0,60 \\
(0,47,0,77)\end{array}$ \\
\hline DECLARE TIMI 58 & Dapagliflozina & $\begin{array}{c}0,93 \\
(0,84,1,03)\end{array}$ & $\begin{array}{c}0,98 \\
(0,82,1,17)\end{array}$ & $\begin{array}{c}0,93 \\
(0,82,1,04)\end{array}$ & $\begin{array}{c}0,73 \\
(0,61,0,88)\end{array}$ & $\begin{array}{c}0,53 \\
(0,43,0,66)\end{array}$ \\
\hline
\end{tabular}

Legenda: CV = Cardiovascular; IC = Insuficiência cardíaca; MACE = Eventos cardiovasculares adversos major. Nota: Adaptado de Home P. Diabetologia 2019. https://doi.org/10.1007/s00125-018-4801.

incluindo diuréticos, a terapia farmacológica com antagonistas dos recetores dos mineralocorticoides deve ser considerada. ${ }^{40}$

\section{Recomendações para terapêutica antidislipidémica}

1. A terapêutica farmacológica com estatina de alta potência (atorvastatina 40-80mg ou rosuvastatina 20$-40 \mathrm{mg}$ ) deve ser considerada na pessoa com DM2. ${ }^{18}$

2. O alvo de LDL na pessoa com DM2 deve situar-se em $<70 \mathrm{mg} / \mathrm{dL}$ nos doentes com RCV alto e $<55 \mathrm{mg} / \mathrm{dL}$ nos casos de RCV muito alto (múltiplos fatores de risco, lesão de órgão-alvo ou DCV estabelecida). ${ }^{41}$

3. Quando o doente com DM2 e DCV estabelecida apresenta um valor de colesterol LDL superior ou igual a $70 \mathrm{mg} / \mathrm{dL}$, estando sob terapêutica com es- tatina na dose máxima tolerada, deve-se considerar a associação com ezetimiba. ${ }^{18}$

4. Até ao momento, os estudos aleatorizados para avaliar o impacto da associação terapêutica entre estatina e fibrato em pessoas com DM2 e de elevado RCV não demonstraram benefício cardiovascular, não sendo por isso uma associação recomendada. ${ }^{18}$

5. A associação farmacológica com estatina e niacina não é recomendada por falta de benefício para o RCV e por se ter verificado aumento do risco de AVC. ${ }^{18}$

Recomendações para terapêutica antitrombótica no doente com DM2

Doentes sem eventos coronários prévios: pré-evento

1. Em doentes com DM2 sem doença coronária 
diagnosticada, o benefício da utilização do AAS em baixa dose é ultrapassado pelo risco hemorrágico, pelo que esta estratégia não é recomendada. ${ }^{27}$

2. O AAS deve ser utilizado como estratégia de prevenção secundária $(100 \mathrm{mg} / \mathrm{dia})$, em doentes com DM2 e doença coronária estável ou DAP concomitante ${ }^{*} ., 27$

3. O AAS deve ainda ser utilizado em doentes com colocação eletiva de stent. Em alternativa, pode ser utilizado clopidogrel $75 \mathrm{mg} /$ dia. $^{8}$

4. Em doentes com DM2 após um AVC é recomendado iniciar o tratamento com AAS dentro das primeiras $48 \mathrm{~h}$ após evento, de forma a prevenir eventos coronários. ${ }^{42}$

\section{Doentes com síndroma coronária aguda: no momen- to do evento e primeiros 12 meses}

1. Em doentes com síndroma coronária aguda, ticagrelor (180mg dose de carga, seguindo-se 90mg bid como dose de manutenção) em conjunto com AAS está recomendado, independentemente da estratégia de tratamento (gestão farmacológica, intervenção coronária percutânea, cirurgia de revascularização do miocárdio), incluindo em doentes previamente tratados com clopidogrel. ${ }^{43}$

2. Em doentes com SCA recomenda-se dupla antiagregação plaquetária (DAPT) por um período de 12 meses, preferencialmente associação de AAS e ticagrelor. ${ }^{43}$

3. Em doentes com síndroma coronária aguda e colocação de stent, caso o risco hemorrágico seja considerado elevado (score PRECISE-DAPT $\geq 25$ ) pode considerar-se descontinuar a terapêutica com DAPT após 6 meses de tratamento (Tabela 3). ${ }^{43}$

\section{Doentes com síndroma coronária aguda: prevenção de novos eventos}

1. Após os primeiros 12 meses é recomendado o tratamento com AAS em baixa dose para todos os doentes. Caso não seja tolerado deve ser considerado clopidogrel como substituição. ${ }^{8}$

2. Independentemente da estratégia de tratamento, doentes que tolerem DAPT sem complicações he-

*Os resultados do estudo THEMIS suportam a dupla antiagregação plaquetária com ticagrelor $60 \mathrm{mg}$ em doentes com DM2 e doença coronária. ${ }^{30}$ morrágicas major (hemorragias fatais, intracranianas ou conducentes a transfusão de sangue ou internamento) durante o primeiro ano podem continuar com DAPT para além desse período. Para avaliar o risco nestes doentes pode ser calculado o Score DAPT aos 12 meses (Tabela 3). ${ }^{42}$

3. No doente com síndroma coronária aguda de elevado risco isquémico sem complicações hemorrágicas, ticagrelor $60 \mathrm{mg}$ bid em associação com AAS pode ser a estratégia preferida para além dos 12 meses, preferencialmente a clopidogrel ou prasugrel..$^{43}$

\section{DISCUSSÃO}

Este documento resume, de forma prática e orientada para o RCV global, a abordagem farmacológica da pessoa com DM2 e suas principais complicações cardiovasculares. Os principais resultados apresentados resumem as recomendações mais adaptadas à prática da medicina geral e familiar, recolhidas das principais orientações clinicas, dos resultados das meta-análises e ensaios clínicos mais recentes e relevantes, e estabelecendo um estado da arte.

A abordagem farmacológica integrada da pessoa com DM2 exige uma visão cuidadosa e holística. Sublinha-se a importância desta abordagem em detrimento da glicocêntrica, com particular atenção o RCV global. É fundamental ultrapassar a inércia terapêutica $\mathrm{e}$ procurar conferir proteção aos múltiplos fatores de risco de forma a melhorar a sua esperança e qualidade de vida futuras. É igualmente importante considerar todas as comorbilidades, sendo que hoje, mais do que nunca, é possível ajustar a terapêutica de forma personalizada e centrada na pessoa.

Tendo em conta que a DM2 afeta de forma adversa diversos aparelhos e sistemas, "the pump (heart failure), pipes (atherosclerosis) and filter (renal disease)", ${ }^{44}$ estão agora à disposição novas armas terapêuticas que prometem proteger todos estes elementos, devendo estas ser consideradas na prática clínica habitual.

Resultados inesperados dos estudos de outcomes cardiovasculares com uma nova classe farmacológica para o controlo da hiperglicemia - os iSGLT2 - revelaram uma nova arma para a IC nestes doentes, trazendo a relação entre IC e DM2 para primeiro plano. Estes estudos demonstraram um efeito robusto na redução dos internamentos por IC e mortalidade cardiovascular. 


\begin{tabular}{|c|c|c|c|}
\hline & \multicolumn{2}{|l|}{ Score DAPT } & Score PRECISE-DAPT \\
\hline Momento de cálculo & \multicolumn{2}{|c|}{ Após 12 meses sem eventos com DAPT } & No momento da angioplastia e colocação do stent \\
\hline $\begin{array}{l}\text { Avaliação de estratégias } \\
\text { de duração de DAPT }\end{array}$ & \multicolumn{2}{|c|}{$\begin{array}{l}\text { DAPT de duração convencional ( } 12 \text { meses) vs } \\
\text { DAPT de duração longa ( } 30 \text { meses) }\end{array}$} & $\begin{array}{l}\text { DAPT curta ( } 3-6 \text { meses) vs } \\
\text { DAPT standard/longa (12-24 meses) }\end{array}$ \\
\hline Cálculo do score & $\begin{array}{l}\text { Idade } \\
\geq 75 \\
65 \text { a }<75 \\
<65 \\
\text { Fumador (cigarros) } \\
\text { Diabetes mellitus } \\
\text { EM no momento da avaliação } \\
\text { IPC ou MI anterior } \\
\text { Stent com eluição de placlitaxel } \\
\text { Diâmetro do stent < 3mm } \\
\text { ICC ou FEVE < 30\% } \\
\text { Implante de stent venoso }\end{array}$ & $\begin{array}{l}\text { Valor } \\
-2 \mathrm{pt} \\
-1 \mathrm{pt} \\
0 \mathrm{pt} \\
+1 \mathrm{pt} \\
+1 \mathrm{pt} \\
+1 \mathrm{pt} \\
+1 \mathrm{pt} \\
+1 \mathrm{pt} \\
+1 \mathrm{pt} \\
+2 \mathrm{pt} \\
+2 \mathrm{pt}\end{array}$ & $\begin{array}{l}\text { Hemoglobina } \\
\text { Contagem de glóbulos brancos } \\
\text { Idade } \\
\text { Clearence de creatinina } \\
\text { Hemorragia prévia } \\
\text { Pontos do score }\end{array}$ \\
\hline Alcance do score & Amplitude & $\begin{array}{l}-2 \text { a } 10 \\
\text { pontos }\end{array}$ & 0-100 pontos \\
\hline $\begin{array}{l}\text { Cut-off de decisão } \\
\text { sugerido }\end{array}$ & \multicolumn{2}{|l|}{$\begin{array}{l}\text { Score } \geq 2 \text { DAPT de duração longa } \\
\text { Score }<2 \text { DAPT de duração } \\
\text { convencional } 12 \text { meses }\end{array}$} & $\begin{array}{l}\text { Score } \geq 25 \text { - DAPT curta } \\
\text { Score }<25 \text { - DAPT standard/longa }\end{array}$ \\
\hline Calculadora & \multicolumn{2}{|l|}{ www.daptstudy.org } & www.precisedaptscore.com \\
\hline
\end{tabular}

Legenda: DAPT = Terapêutica com dupla antiagregação plaquetar.

A nefropatia diabética é uma complicação grave que surge em cerca de 20 a $40 \%$ de todos os doentes com DM2 ${ }^{45}$ Por outro lado, a doença renal é um fator de risco independente para doença cardiovascular, associando-se à constelação de fatores que se juntam em torno da pessoa com DM2. Os inibidores do SGLT2 demostraram igualmente reduzir de forma significativa a progressão da doença renal crónica em doentes com DM2, mesmo em doentes já com tratamento otimizado. Esta classe farmacológica, juntamente com alguns dos arGLP-1, mostraram também reduzir a incidência de complicações ateroscleróticas.

Há duas décadas que a prática estabelecida implicava a utilização de antiagregação simples com aspirina nos doentes com DM2, considerando o conceito de equivalente de doença cardiovascular estabelecida. Estudos ao longo dos últimos anos foram recomendando a utilização de aspirina apenas nos doentes com DM e com RCV mais elevado. Mais recentemente, dois estudos em 2018 - ASCEND ${ }^{27}$ e ARRIVE ${ }^{46}$ - revisitaram o papel da aspirina como estratégia de prevenção primária de eventos cardiovasculares nos doentes com DM2, mostrando que o efeito benéfico da aspirina em termos de redução de eventos isquémicos suporta um importante custo em termos de complicações hemorrágicas, não se equilibrando a balança a favor de uma relação custo-benefício favorável. Os efeitos farmacológicos da aspirina não mudaram ao longo dos anos, mas seguramente mudaram as populações em estudo, uma vez que na era atual os doentes estão melhor tratados (diagnóstico mais precoce, melhor controlo glicémico, tensional e da dislipidemia) e o seu risco remanescente será provavelmente menor. A aspirina continua, contudo, a demonstrar um claro benefício como estratégia 
de prevenção secundária, permanecendo como pedra basilar no tratamento do doente com doença aterosclerótica estabelecida.

$\mathrm{Na}$ abordagem da dislipidemia, contudo, não se registaram grandes mudanças nos últimos anos, pelo menos em termos de recomendações terapêuticas e de novos fármacos. $\mathrm{O}$ foco continua a estar centrado na mudança de estilo de vida e os fármacos de primeira linha para o controlo da dislipidemia continuam a ser as estatinas. O mesmo ocorre na terapêutica da hipertensão, apesar de existir alguma evidência recente sugerindo que a toma de um fármaco anti-hipertensor ao deitar possa ter benefícios no doente com DM2. Esta abordagem centrada em princípios de crenoterapia tem sido ainda controversa, pelo que ainda não há recomendações formais nesse sentido. ${ }^{18,47}$

Apesar de a diabetes ser abordada e gerida por inúmeras espacialidades médicas, fruto da sua complexidade e abrangência, o médico de família é o profissional que tem mais facilidade em ter uma visão de conjunto, podendo abordar a totalidade das áreas nas quais a DM2 pode trazer complicações e gerir a prestação de cuidados de outros problemas de saúde em simultâneo. Por outro lado, acompanha de perto estes doentes, contactando com eles de forma frequente. Neste sentido, exorta-se os médicos de família a assumir o papel central e aglutinador entre os vários especialistas que cuidam da pessoa com DM2. O médico de família, enquanto elemento central, será fundamental na escolha e na revisão contínua da terapêutica para controlo da hiperglicemia, na decisão sobre qual a melhor terapêutica antiagregante, no controlo da dislipidemia e da hipertensão arterial. A integração das particularidades e preferências da pessoa em tratamento é fundamental para garantir o seu envolvimento no plano terapêutico de forma continuada, sendo um fator determinante na manutenção da qualidade de vida.

Espera-se que este documento permita ao clínico encontrar as respostas a cada uma das questões seguintes, que surgirão sempre que o médico se pergunte se o doente que tem à sua frente está bem tratado:

- O doente está bem controlado do ponto de vista glicémico? Isto é, atinjo o meu alvo, com o maior benefício e o menor risco de efeitos secundários ou iatrogénicos? Estou a maximizar a capacidade de prevenir DCV aterosclerótica? Estou a maximizar a ca- pacidade de prevenir a progressão da IC ou da DRC?

- O doente tem a sua pressão arterial bem controlada?

- Há evidência de doença renal (albuminúria ou diminuição da taxa de filtração glomerular)? Está medicado com fármacos modificadores do eixo renina-angiotensina? Há lugar a uso de um fármaco para controlo da hiperglicemia que ofereça proteção renal?

- Como está a ficha lipídica do doente e qual o seu impacto no seu perfil de risco? Está medicado com estatina? Precisará de ser intensificada a terapêutica?

- Haverá indicação para antiagregação plaquetária? Pelo seu perfil de comorbilidades, haverá indicação para antiagregação simples? Ou necessita de terapêutica dupla face a um prévio evento coronário?

A abordagem farmacológica deve ser, assim, integrada, permitindo otimizar os benefícios terapêuticos, minimizando o risco para o doente e individualizando a abordagem ao seu perfil clínico e pessoal, bem como preferências, crenças e estilo de vida.

\section{CONCLUSÃO}

Este trabalho de revisão, abordando os diversos aspetos a considerar na pessoa com DM2, salienta a importância que esta doença apresenta ao envolver diversos aparelhos e sistemas, para a qual a medicina geral e familiar está direcionada, uma vez que, pela sua abrangência e especificidade, é a melhor posicionada para a gestão completa do problema.

O médico de família assume, assim, um papel fundamental na prestação de cuidados a estas pessoas, uma vez que tem uma visão de conjunto da pessoa, da sua doença, das comorbilidades e complicações. O médico de família é o especialista mais próximo destes doentes e, como tal, encontra-se numa posição privilegiada para integrar essa visão aos desafios da pessoa em si, às suas preferências pessoais, ambições e expectativas, ao seu estilo de vida, integrando, deste modo, o melhor balanço entre benefício e risco das terapêuticas instituídas.

Novos dados surgirão no futuro relativamente à terapêutica da DM2 e suas comorbilidades e complicações. Estão em curso estudos com novas classes e novos medicamentos que terão o potencial de abordar as complicações micro e macrovasculares como antes não tinha sido conseguido, nomeadamente a insuficiência 
cardíaca ou a progressão da lesão renal. Poder-se-á avizinhar, então, uma era em que a morbimortalidade associada à DM2 poderá começar a ser verdadeiramente combatida na sua génese e, com isto, melhorar a qualidade de vida das pessoas.

O médico de família deverá estar na vanguarda deste conhecimento, assumindo-se na linha da frente para a gestão integrada da pessoa com DM2.

\section{AGRADECIMENTOS}

Nada a referir.

\section{REFERÊNCIAS BIBLIOGRÁFICAS}

1. Observatório Nacional da Diabetes. Diabetes: factos e números - O ano de 2015. Lisboa: Sociedade Portuguesa de Diabetologia; 2016. ISBN 9789899666320

2. Direção-Geral da Saúde. Programa nacional para a diabetes. Lisboa: DGS; 2017.

3. Adler Al, Stratton IM, Neil HA, Yudkin JS, Matthews DR, Cull CA, et al. Association of systolic blood pressure with macrovascular and microvascular complications of type 2 diabetes (UKPDS 36): prospective observational study. BMJ. 2000;321(7258):412-9.

4. Roger VL, Go AS, Lloyd-Jones DM, Adams RJ, Berry JD, Brown TM, et al. Heart disease and stroke statistics - 2011 update: a report from the American Heart Association. Circulation. 2011;123(4):e18-e209.

5. Donahoe SM, Stewart GC, McCabe CH, Mohanavelu S, Murphy SA, Cannon $\mathrm{CP}$, et al. Diabetes and mortality following acute coronary syndromes. JAMA. 2007;298(7):765-75.

6. Ritz E, Orth SR. Nephropathy in patients with type 2 diabetes mellitus. N Engl J Med. 1999;341(15):1127-33.

7. Ahmad FS, Ning H, Rich JD, Yancy CW, Lloyd-Jones DM, Wilkins JT. Hypertension, obesity, diabetes, and heart failure-free survival: the Cardiovascular Disease Lifetime Risk Pooling Project. JACC Heart Fail. 2016;4(12):911-9.

8. Montalescot G, Sechtem U, Achenbach S, Andreotti F, Arden C, Budaj A, et al. 2013 ESC guidelines on the management of stable coronary artery disease: the Task Force on the management of stable coronary artery disease of the European Society of Cardiology. Eur Heart J. 2013;34(38):2949-3003.

9. Aboyans V, Ricco JB, Bartelink ML, Björck M, Brodmann M, Cohnert T, et al. 2017 ESC guidelines on the diagnosis and treatment of peripheral arterial diseases, in collaboration with the European Society for Vascular Surgery (ESVS): document covering atherosclerotic disease of extracranial carotid and vertebral, mesenteric, renal, upper and lower extremity arteries. Endorsed by: the European Stroke Organization (ESO), the Task Force for the Diagnosis and Treatment of Peripheral Arterial Diseases of the European Society of Cardiology (ESC) and of the European Society for Vascular Surgery (ESVS). Eur Heart J. 2018;39(9): 763-816.

10. KannelWB, McGee DL. Diabetes and cardiovascular risk factors: the Framingham study. Circulation. 1979;59(1):8-13.

11. Haffner SM, Lehto S, Rönnemaa T, Pyörälä K, Laakso M. Mortality from coronary heart disease in subjects with type 2 diabetes and in nondiabetic subjects with and without prior myocardial infarction. N Engl J Med. 1998;339(4):229-34.

12. Nichols GA, Gullion CM, Koro CE, Ephross SA, Brown JB. The incidence of congestive heart failure in type 2 diabetes: an update. Diabetes Care. 2004;27(8):1879-84.

13. Duarte R, Melo $M$, Silva Nunes J, et al. Recomendações nacionais da SPD para o tratamento da hiperglicemia na diabetes tipo 2: atualização 2018/19 com base na posição conjunta ADA/EASD [SPD national recommendations for the treatment of hyperglycemia in type 2 diabetes: update based in the ADA/EASD Joint Position Statement]. Rev Port Diabetes. 2018;13(4):154-80. Portuguese

14. DeFronzo RA. From the triumvirate to the ominous octet: a new paradigm for the treatment of type 2 diabetes mellitus. Diabetes. 2009;58(4):773-95.

15. Holman RR, Paul SK, Bethel MA, Matthews DR, Neil AW. 10-Year follow-up of intensive glucose control in type 2 diabetes. N Engl J Med. 2008;359(15):1577-89.

16. Zelniker TA, Wiviott SD, Raz I, Im K, Goodrich EL, Bonaca MP, et al. SGLT2 inhibitors for primary and secondary prevention of cardiovascular and renal outcomes in type 2 diabetes: a systematic review and meta-analysis of cardiovascular outcome trials. Lancet. 2019;393(10166):31-9.

17. Bethel MA, Patel RA, Merrill P, Lokhnygina Y, Buse JB, Mentz RJ, et al. Cardiovascular outcomes with glucagonlike peptide 1 receptor agonists in patients with type 2 diabetes: a metaanalysis. Lancet Diabetes Endocrinol. 2018;6(2):105-13.

18. American Diabetes Association. 10. Cardiovascular disease and risk management: standards of medical care in diabetes-2019. Diabetes Care. 2019;42(Suppl 1):S103-23.

19. Davies MJ, D'Alessio DA, Fradkin J, Kernan WN, Mathieu C, Mingrone $\mathrm{G}$, et al. Management of hyperglycemia in type 2 diabetes, 2018: a consensus report by the American Diabetes Association (ADA) and the European Association for the Study of Diabetes (EASD). Diabetes Care. 2018;41(12):2669-701.

20. Ivers NM, Jiang M, Alloo J, Singer A, Ngui D, Casey CG, et al. Diabetes Canada 2018 clinical practice guidelines: key messages for family physicians caring for patients living with type 2 diabetes. Can Fam Physician. 2019;65(1):14-24.

21. Zinman B, Wanner C, Lachin JM, Fitchett D, Bluhmki E, Hantel S, et al. Empagliflozin, cardiovascular outcomes, and mortality in type 2 diabetes. N Engl J Med. 2015;373(22):2117-28.

22. Neal B, Perkovic V, Mahaffey KW, de Zeeuw D, Fulcher G, Erondu N, et al. Canagliflozin and cardiovascular and renal events in type 2 diabetes. N Engl J Med. 2017;377(7):644-57.

23. Wiviott SD, Raz I, Bonaca MP, Mosenzon O, Kato ET, Cahn A, et al. Dapagliflozin and cardiovascular outcomes in type 2 diabetes. N Engl J Med. 2019;380(4):347-57.

24. Pfeffer MA, Claggett B, Díaz R, Dickstein K, Gerstein HC, Køber LV, et al. Lixisenatide in patients with type 2 diabetes and acute coronary syndrome. N Engl J Med. 2015;373(23):2247-57.

25. Marso SP, Daniels GH, Brown-Frandsen K, Kristensen P, Mann JF, Nauck $M A$, et al. Liraglutide and cardiovascular outcomes in type 2 diabetes. N Engl J Med. 2016;375(4):311-22.

26. Holman RR, Bethel MA, Mentz RJ, Thompson VP, Lokhnygina Y, Buse JB, 
et al. Effects of once-weekly exenatide on cardiovascular outcomes in type 2 diabetes. N Engl J Med. 2017;377(13):1228-39.

27. ASCEND Study Collaborative Group, Bowman L, Mafham M, Wallendszus K, Stevens W, Buck G, et al. Effects of aspirin for primary prevention in persons with diabetes mellitus. N Engl J Med. 2018;379(16) 1529-39.

28. McNeil JJ, Wolfe R, Woods RL, Tonkin AM, Donnan GA, Nelson MR, et al. Effect of aspirin on cardiovascular events and bleeding in the healthy elderly. N Engl J Med. 2018;379(16):1509-18.

29. Bonaca MP, Bhatt DL, Cohen M, Steg PG, Storey RF, Jensen EC, et al. Long-term use of ticagrelor in patients with prior myocardial infarction. N Engl J Med. 2015;372(19):1791-800.

30. Steg PG, Bhatt DL, Simon T, Fox K, Mehta SR, Harrington RA, et al. Ticagrelor in patients with stable coronary disease and diabetes. N Eng J Med. 2019;381(14):1309-20.

31. Arnett DK, Blumenthal RS, Albert MA, Buroker AB, Goldberger ZD, Hahn EJ, et al. 2019 ACC/AHA guideline on the primary prevention of cardiovascular disease: a report of the American College of Cardiology/ /American Heart Association Task Force on Clinical Practice Guidelines. Circulation. 2019;140(11):e596-e646.

32. Fried LP, Tangen CM, Walston J, Newman AB, Hirsch C, Gottdiener J, et al. Frailty in older adults: evidence for a phenotype. J Gerontol A Biol Sci Med Sci. 2001;56(3):M146-56.

33. Furtado RH, Bonaca MP, Raz I, Zelniker TA, Mosenzon O, Cahn A, et al. Dapagliflozin and cardiovascular outcomes in patients with type 2 diabetes mellitus and previous myocardial infarction. Circulation. 2019; 139(22):2516-27.

34. Kato ET, Silverman MG, Mosenzon O, Zelniker TA, Furtado RH, et al. Effect of dapagliflozin on heart failure and mortality in type 2 diabetes mellitus. Circulation. 2019;139(22):2528-36.

35. Perkovic V, Jardine MJ, Neal B, Bompoint S, Heerspink HJ, Charytan DM, et al. Canagliflozin and renal outcomes in type 2 diabetes and nephropathy. N Engl J Med. 2019;380(24):2295-306.

36. Wanner C, Inzucchi SE, Lachin JM, Fitchett D, von Eynatten M, Mattheus $M$, et al. Empagliflozin and progression of kidney disease in type 2 diabetes. N Engl J Med. 2016;375(4):323-34.

37. Fioretto P, Del Prato S, Buse JB, Goldenberg R, Giorgino F, Reyner D, et al. Efficacy and safety of dapagliflozin in patients with type 2 diabetes and moderate renal impairment (chronic kidney disease stage 3A): the DERIVE study. Diabetes Obes Metab. 2018;20(11):2532-40.

38. American Diabetes Association. 11. Microvascular complications and foot care: standards of medical care in diabetes-2019. Diabetes Care. 2019;42(Suppl 1):S124-38.

39. Pan F, Chernew ME, Fendrick AM. Impact of fixed-dose combination drugs on adherence to prescription medications. J Gen Intern Med. 2008;23(5):611-4.

40. Williams B, Mancia G, Spiering W, Rosei EA, Azizi M, Burnier M, et al. 2018 ESC/ESH guidelines for the management of arterial hypertension. Eur Heart J. 2018;39(33):3021-104.

41. Mach F, Baigent C, Catapano AL, Koskinas KC, Casula M, Badimon L, et al. ESC/EAS guidelines for the management of dyslipidaemias: lipid modification to reduce cardiovascular risk. Eur Heart J. 2020;41(1):11188.
42. Powers WJ, Rabinstein AA, Ackerson T, Adeoye OM, Bambakidis NC, Becker K, et al. 2018 guidelines for the early management of patients with acute ischemic stroke: a guideline for healthcare professionals from the American Heart Association/American Stroke Association. Stroke. 2018;49(3):e46-e99.

43. Valgimigli M, Bueno H, Byrne RA, Collet JP, Costa F, Jeppsson A, et al. 2017 ESC focused update on dual antiplatelet therapy in coronary artery disease developed in collaboration with EACTS: the Task Force for dual antiplatelet therapy in coronary artery disease of the European Society of Cardiology (ESC) and of the European Association for Cardio-Thoracic Surgery (EACTS). Eur Heart J. 2018;39(3):213-60.

44. Verma S, Jüni $P$, Mazer CD. Pump, pipes, and filter: do SGLT2 inhibitors cover it all? Lancet. 2019;393(10166):3-5.

45. Centers for Disease Control and Prevention. National diabetes statistics report [homepage]. Washington: CDC; 2019 [updated 2020 Feb 14; cited 2019 Jun 18].Available from: https://www.cdc.gov/diabetes/data/ statistics/statistics-report.html

46. Gaziano JM, Brotons C, Coppolecchia R, Cricelli C, Darius H, Gorelick $\mathrm{PB}$, et al. Use of aspirin to reduce risk of initial vascular events in patients at moderate risk of cardiovascular disease (ARRIVE): a randomised, double-blind, placebo-controlled trial. Lancet. 2018;392 (10152):1036-46.

47. Hermida RC, Crespo JJ, Domínguez-Sardiña M, Otero A, Moyá A, Ríos MT, et al. Bedtime hypertension treatment improves cardiovascular risk reduction: the Hygia Chronotherapy Trial. Eur Heart J. 2019;ehz754.

\section{CONFLITO DE INTERESSES}

Os autores declaram o apoio logístico e financeiro da companhia AstraZeneca@. O trabalho foi elaborado com total isenção científica por parte dos autores. A companhia AstraZeneca@ não teve influência sobre os conteúdos ou julgamentos técnico-científicos na elaboração das recomendações aqui publicadas.

Manuel Rodrigues Pereira declara ainda que, entre 2015 e 2017, integrou o Departamento Médico da Janssen e que, desde 2018, recebeu honorários como prestador de serviços ou teve apoio para participação em congressos e eventos similares das seguintes empresas que trabalham na área da diabetes mellitus: AstraZeneca (no âmbito da elaboração deste artigo); Boehringer-Ingelheim; Eli Lilly and Company; NovoNordisk; e Tecnifar.

\section{FINANCIAMENTO}

A elaboração deste trabalho foi financiada pela companhia AstraZeneca@ na cobertura das despesas logísticas e operacionais inerentes. AAstraZeneca não influenciou o conteúdo da publicação.

\section{ENDEREÇO PARA CORRESPONDÊNCIA}

Tiago Maricoto

E-mail: tiago.maricoto@gmail.com

https://orcid.org/0000-0002-4201-9565

Recebido em 06-11-2019

Aceite para publicação em 15-03-2020 


\section{ABSTRACT}

\section{AURORA CONSENSUS: CARDIOVASCULAR RISK OF TYPE 2 DIABETIC PATIENTS}

Objective: To develop a consensus document, guided to general and family medicine clinical practice that addresses the key aspects of the drug management on type 2 diabetes and its cardiovascular risk.

Methods: An expert panel composed of six family doctors undertaken a non-systematic literature search and review, gathering the main international clinical guidelines and the most relevant meta-analysis, clinical trials and reviews, and regarding the management of type 2 diabetes and its main cardiovascular outcomes. The selected references were further and exhaustively analysed and then summarized on brief statements for clinical practice.

Results: We identified 23 different bibliographic references, including guidelines, meta-analysis, and clinical trials, from which a set of statements were developed, namely 13 statements for diabetic treatment, five for hypertension treatment, five for dyslipidaemia therapy and 10 for anti-platelet therapy.

Conclusion: The management of type 2 diabetes and its main cardiovascular outcomes must be personalized at an individual level, a challenge that requires a comprehensive understanding. New drug therapies are now available, and they must be used in an incisive way to ensure good clinical control of type 2 diabetes and its further cardiovascular outcomes, and thus, reducing the risk for additional comorbidities.

Keywords: Type 2 diabetes; General practice; Family medicine; Cardiovascular risk. 